\title{
The establishment of supramolecular immunobead real-time PCR and the identification of Cox-2 as a metastasis-related marker in colorectal carcinoma
}

\author{
ZU-GUO LI*, XIAO-YAN WANG ${ }^{*}$, JIAN-LAN CHANG ${ }^{*}$, WEI-BING XIE, \\ TENG-FEI LIU, QING-LING ZHANG, YONG-JIAN DENG and YAN-QING DING
}

Department of Pathology, Nanfang Hospital, Southern Medical University; Department of Pathology, School of Basic Medical Sciences, Southern Medical University, Guangzhou 510515, P.R. China

Received May 4, 2012; Accepted May 30, 2012

DOI: $10.3892 /$ or.2012.1867

\begin{abstract}
Cyclooxygenase-2 (Cox-2) is an inducible enzyme that converts arachidonic acid to prostaglandins, and it is hypothesized to induce carcinogenesis and metastasis in colorectal cancer. Our previous data also indicated that a higher expression level of Cox-2 was correlated with colorectal cancer metastasis. The Cox-2 protein was detected in the glandular cavity of colorectal cancer and the surrounding interstitial tissues. The usefulness of the Cox-2 gene as a gene therapy target and diagnostic marker remains unknown. In this study, a method using immuno-PCR and real-time PCR followed by supramolecular immunobead real-time PCR was established and used to detect the expression of Cox-2 in serum samples of nude mice with colorectal carcinoma. In addition, we established a Cox-2 gene stable knockdown colorectal cell line (SW480-EGFP-Cox-2 shRNA) using lentiviral vector-mediated RNA interference (RNAi) technology and established an imageable colorectal cancer metastasis mouse model. We found that the proliferation, invasion and tumorigenesis of SW480-EGFP-Cox-2 shRNA cells were attenuated compared with SW480 cells. In vivo experiments also demonstrated that angiogenesis in the Cox-2 knockdown colorectal cancer cells was decreased. The whole body optical imaging revealed that the SW480-EGFP-Cox-2 shRNA cells had an abrogated ability to develop metastases in the lymph nodes, lungs or liver in vivo. The improved immunobead PCR assay detected significantly lower Cox-2 protein levels in the serum samples of the SW480-EGFP-Cox-2 shRNA group compared with those of the SW480-EGFP-Cox-2-Ctrl shRNA group. In conclusion, our results indicated that the knockdown
\end{abstract}

Correspondence to: Dr Yan-Qing Ding, Department of Pathology, Nanfang Hospital, Southern Medical University, Guangzhou 510515, P.R. China

E-mail: dyqgz@126.com

*Contributed equally

Key words: Cox-2, colorectal cancer, gene therapy, target gene, metastasis, serum marker, immuno-PCR of Cox-2 expression suppressed the proliferation and invasion of colorectal cancer cells both in vitro and in vivo. This study also demonstrated that silencing Cox-2 in vivo reduced the metastastic potential of colorectal cancer. Thus, Cox-2 is a promising marker for the diagnosis of colorectal metastasis and a potential therapeutic target for colorectal cancer.

\section{Introduction}

Colorectal cancer is the third leading cause of cancer-related death in the world and is a multistep and multigene process. Several studies have shown that the aberrant expression of the cyclooxygenase-2 (Cox-2) gene is related to colorectal cancer $(1,2)$. Cox-2 is an inducible enzyme that converts arachidonic acid to prostaglandins. Through the production of prostaglandins, Cox-2 induces carcinogenesis by promoting cell proliferation, inhibiting apoptosis, stimulating angiogenesis, and mediating immune suppression (3-8). Studies have revealed that the Cox- 2 gene is upregulated in human colorectal adenomas and adenocarcinoma $(9,10)$. Our previous studies found that a high expression level of Cox-2 is related to the metastasis of colorectal cancer. Interestingly, we observed that Cox-2 was expressed in the glandular cavity of colorectal cancer and in the surrounding interstitial tissues (11). The usefulness of the Cox-2 protein as a colorectal carcinoma diagnostic marker needs to be explored. Non-steroidal anti-inflammatory drugs (NSAIDs) could inhibit the enzymatic activity of Cox-2 and suppress the growth of cancer cells (12-17). Furthermore, the long-term use of NSAIDs was associated with a reduction in colorectal cancer risk. In animal models, Cox-2 inhibitors can reduce the incidence of colon cancer in APC knockout mice treated with chemical carcinogens (18). However, more studies are needed to demonstrate whether the Cox-2 gene can be used as a colorectal carcinoma gene therapy target.

In this study, we sought to establish an imageable metastasis model of colorectal cancer and to evaluate whether the Cox-2 gene can be used as a gene therapy target for colorectal cancer. A new, improved immunobead-PCR technique was used to detect Cox-2 in the serum using the imageable metastasis model of colorectal cancer to evaluate whether serum Cox-2 is a useful diagnostic serum marker. 
Table I. The Cox-2 specific shRNA sequences.

\begin{tabular}{ll}
\hline shRNA & \multicolumn{1}{c}{ Sequence } \\
\hline shRNA1 & 5'-CACCGCTGGGAAGCCTTCTCTAACGAATTAGAGAAGGCTTCCCAGC-3' \\
shRNA2 & 5'-AAAAGCTGGGAAGCCTTCTCTAATTCGTTAGAGAAGGCTTCCCAGC-3' \\
& 5'-CACCGCTTTATGCTGAAGCCCTACGAATAGGGCTTCAGCATAAAGC-3' \\
shRNA3 & 5'-AAAAGCTTTATGCTGAAGCCCTATTCGTAGGGCTTCAGCATAAAGC-3' \\
& 5'-CACCGCTGTCCCTTTACTTCATTCGAAAATGAAGTAAAGGGACAGC-3' \\
Ctrl-shRNA & 5'-AAAAGCTGTCCCTTTACTTCATTTTCGAATGAAGTAAAGGGACAGC-3' \\
& 5'-CACCGCTACCTTCGCCCATATGGAATTTTGCCGACCAAACGGAGC-3' \\
& 5'-AAAAGCTCCGTTTGGTACGGCAAAATTCCATATGGGCGAAGGTAGC-3'
\end{tabular}

\section{Materials and methods}

Cell lines and animals. The human colorectal cancer cell line SW480 was purchased from the American Type Culture Collection (ATCC). The human colorectal cancer cell line SW480/EGFP, which stably expresses the EGFP protein, was established from the SW480 cells by the transfection of the pEGFP-N1 plasmid and was cultured in RPMI-1640 medium (Gibco) supplemented with $10 \%$ fetal bovine serum (FBS) and $100 \mathrm{U} / \mathrm{ml}$ penicillin/streptomycin in a $5 \% \mathrm{CO}_{2}$ humidified atmosphere at $37^{\circ} \mathrm{C}$.

The 293FT cells were cultured in Dulbecco's modified Eagle's medium (Gibco). Four-week-old female/male athymic BALB/c nu/nu mice were purchased from the Central Laboratory of Animal Science at Southern University and maintained in laminar-flow cabinets under specific pathogenfree conditions.

Preparation of lentiviral vectors. Three different shRNA sequences targeting the Cox-2 gene were selected using the Block-iT ${ }^{\mathrm{TM}}$ RNAi Designer (Invitrogen, Carlsbad, CA) (http://rnaidesigner.invitrogen.com/rnaiexpress/rnaiExpress. jsp) (Table I). The human Cox-2 short hairpin RNA (shRNA) lentiviral-expressing vectors were used with the Block-iT ${ }^{\mathrm{TM}}$ Lentiviral RNAi Expression system (catalog no. K4944-00; Invitrogen) following the manufacturer's instructions. In brief, 3 pLenti6/Cox-2 shRNA expression vectors containing the human Cox-2 shRNA-expressing cassette and 1 scramble control vector were constructed. The replication-incompetent lentivirus was produced by cotransfection of the pLenti6/ Cox-2 shRNA expression vector and ViraPower packaging mix (Invitrogen) containing an optimized mixture of three packaging plasmids: pLP1, pLP2 and pLP/VSVG, into 293FT cells. The viral supernatant was harvested $48 \mathrm{~h}$ after transfection, filtered through a $0.45-\mu \mathrm{m}$ cellulose acetate filter, and frozen at $-70^{\circ} \mathrm{C}$.

Construction of the stable Cox-2-silencing cell line. The SW480/EGFP cells were transduced with the most effective Cox-2 shRNA lentiviral vectors or the negative control lentiviral vectors. The cells were selected for stable integration by culturing in complete medium containing blasticidin.
Real-time PCR. The total RNA was extracted using TRIzol reagent (Invitrogen, Carlsbad, CA, USA), and the cDNA was synthesized using SuperScript reverse transcriptase (Takara). The real-time PCR was performed using a Mx3000P real-time PCR system (Stratagene, La Jolla, CA) and Brilliant SYBRGreen QPCR Master Mix kit (Stratagene) following the manufacturer's protocol. The human Cox-2 and GAPDH primers were as follows: Cox-2, forward primer: 5'-AAGTCC CTGAGCATCTACG-3' and the reverse primer: 5'-TTCCTAC CACCAGCAACC-3'. GAPDH, the forward primer: 5'-ATC TCTGCCCCCTCTGCTGA-3' and reverse primer: 5'-GATG ACCTTGCCCACAGCCT-3'.

Western blot analysis. The cells were washed twice with cold phosphate-buffered saline (PBS) and lysed on ice in RIPA buffer with an added cocktail of protease inhibitors. The protein lysates were resolved on a $10 \%$ SDS polyacrylamide gel, electrotransferred to PVDF membranes (Millipore) and blocked in 5\% non-fat dry milk in Tris-buffered saline. The membranes were immunoblotted overnight at $4^{\circ} \mathrm{C}$ with an anti-Cox-2 monoclonal antibody (Sigma) or an anti-tubulin antibody (Sigma) followed by their respective horseradish peroxidase-conjugated secondary antibodies. The signals were detected by enhanced chemiluminescence (Pierce).

Immunocytochemistry. The cells were plated on a cover glass slide in 6-well plates with medium for $24 \mathrm{~h}$ until cells were $30-50 \%$ confluent. The cells on the cover glass were fixed with cold methanol for $2 \mathrm{~min}$ and incubated for $1 \mathrm{~h}$ with the Cox-2 primary antibody (Sigma) at $37^{\circ} \mathrm{C}$. The cells were then incubated with the secondary antibody (goat anti-mouse IgG conjugated to peroxidase, Dingguo, China) for $1 \mathrm{~h}$ at $37^{\circ} \mathrm{C}$. The cells were DAB substrate stained and then counterstained with hematoxylin for $10 \mathrm{sec}$. All of the steps were interspersed by rigorous rinsing with PBS 3 times. The staining was evaluated under a microscope.

MTT assay. The cells were seeded into 96-well culture plates at $1 \times 10^{3}$ cells per well. After they were cultured for 24,48 or $72 \mathrm{~h}$, the cells in each well were incubated with $20 \mu 1$ of $5 \mathrm{mg} / \mathrm{ml}$ 3-(4,5-dimethylthiazol-2-yl)-2,5-diphenyltetrazolium bromide (MTT, Promega) for $4 \mathrm{~h}$. The supernatant was removed, and $150 \mu$ l of dimethyl sulfoxide (DMSO Sigma) was added and 
incubated for $15 \mathrm{~min}$. The absorbance value (OD) of each well was measured using a microplate reader set at $570 \mathrm{~nm}$. All of the experiments were performed in triplicate.

Plate clone formation assay. Approximately $1 \times 10^{2}$ cells were added to each well of a 6 -well culture plate, and each group contained three wells. Following incubation at $37^{\circ} \mathrm{C}$ for 12 days, the cells were washed twice with PBS and stained with Giemsa solution. The number of colonies containing $\geq 50$ cells was counted under a microscope. The plate clone formation efficiency $=$ (number of colonies/number of cells inoculated) $\mathrm{x} 100 \%$. All of the experiments were performed in triplicate.

Invasion assay. Warm serum-free medium was added to the top chamber of the cell invasion chamber (Chemicon) to rehydrate the Matrigel layer for $2 \mathrm{~h}$ at room temperature. A total of $1 \times 10^{5}$ cells were seeded in the top chamber in serum-free medium (300 $\mu$ l containing $1 \times 10^{5}$ cells). The bottom chamber was prepared with $10 \%$ FBS as a chemoattractant. After a 48-h incubation, the membrane were fixed with methanol and stained with hematoxylin. The cells were counted under a microscope in five predetermined fields at $\times 200$. All of the experiments were performed in triplicate.

In vivo metastasis assays. The method of colon surgical orthotopic implantation was previously described (19).

Animal experiments were conducted in accordance with the Animal Research Committee Guidelines of Southern Medical University [SCXK (Yue) 2006-0015, 2006B023]. The whole-body optical images (Lighttools, Encinitas) were used to observe the real-time primary tumor growth and the formation of metastatic lesions. Two months later, before the mice were euthanized, blood samples from all of the nude mice were collected. The blood samples were immediately frozen at $-80^{\circ} \mathrm{C}$ for analysis. All of the mice were then euthanized, individual organs were excised, and metastases were checked by hematoxylin-eosin (H\&E) staining.

Immuno-PCR assay. The Cox-2 protein in the serum samples of the metastasis models of colorectal carcinoma were detected using our new, improved immuno-PCR assay. The assay for detecting very low concentrations of Cox-2 protein in the serum is described as follows (Fig. 5A). First, biotinylated 289 bp reporter DNA fragment originating from the pEGFP-N1 plasmid was amplified using PCR with the following primers: forward primer, biotin-5'-CAGTGCTT CAGCCGCTACCC-3', and reverse primer, biotin-5'-AGTT CACCTTGATGCCGTTCTT-3' (Sangon, Shanghai). The PCR product was purified using a QIAquick PCR purification kit (Qiagen). In the second step, the preparation of reporter DNA-protein conjugates was performed. This reporter DNA-protein conjugate was made by conjugating recombinant streptavidin and the biotinylated dsDNA fragment and biotinylated antibody (goat anti-mouse IgG, Sigma). The biotinylated DNA $(1 \mu \mathrm{M})$ was mixed with $1 \mu \mathrm{M}$ recombinant streptavidin (Roche) and $1 \mu \mathrm{M}$ biotinylated antibody in Trisbuffered saline, incubated for $30 \mathrm{~min}$ at room temperature and filtered and purified using Pall Microsep 100K ultrafiltration equipment (with Omega PES membrane inside, Millipore). The purified reporter DNA-protein conjugates were frozen at $-20^{\circ} \mathrm{C}$ until further analysis. This complex can be conjugated to antigen and captured by immunobeads to be used as the template for PCR.

Third, signal amplification was performed by conventional PCR. The Dynabeads M-280 immunobead (labeled by sheep anti-rabbit IgG, Dynal), rabbit anti-Cox-2 antibody (Sigma), serum samples and mouse anti-Cox-2 antibody (Sigma) were added into PCR tubes in this order. The Cox- 2 protein in the serum samples will be captured by the immunobeads and the Cox-2 antibody. The PCR tubes were thoroughly washed with PBS by magnetic force rinse on the Dynal magnetic platform. The super-molecule complex was then added. The antibody in the complex (biotinylated goat anti-mouse $\mathrm{IgG}$, Sigma) combines with the mouse anti-Cox-2 antibody, which is bound to the serum Cox-2 protein specifically. The reporter DNA in the complex serves as the amplification template of immuno-bead PCR. The complex was then washed in the PCR tube repeatedly by magnetic force rinse to remove the uncombined impurities. Conventional PCR was performed using a thermocycler (Biometra). The PCR mixture contained $3 \mu \mathrm{l}$ of the template DNA, $200 \mu \mathrm{M}$ of each deoxynucleoside triphosphate, $15 \mathrm{pmol}$ of the primers designed for the reporter DNA (forward primer: 5'-CAGTGCTTCAGCCGCTACCC-3', reverse primer: 5'-AGTTCACCTTGATGCCGTTCTT-3'), $3 \mu \mathrm{l}$ of 10 -fold-concentrated polymerase synthesis buffer, $1.5 \mathrm{mM} \mathrm{MgCl}_{2}$, and $0.65 \mathrm{U}$ of Taq DNA polymerase. The thermal cycling conditions were as follows: $95^{\circ} \mathrm{C}$ for $5 \mathrm{~min}$ and 30 cycles at $95^{\circ} \mathrm{C}$ for $30 \mathrm{sec}$, followed by $56^{\circ} \mathrm{C}$ for $45 \mathrm{sec}$ and $72^{\circ} \mathrm{C}$ for $60 \mathrm{sec}$. Aliquots $(15 \mu \mathrm{l})$ of the PCR products were analyzed by electrophoresis on a $1.5 \%$ agarose gel and visualized using ethidium bromide staining. The positive controls and negative controls were performed simultaneously. Identical 289 bp DNA bands in the samples and the positive controls, but not in the negative controls, indicated that there was Cox-2 protein in the serum samples.

Statistical analysis. The SPSS software package, version 13.0 (Abbott Laboratories), was used to perform the statistical comparisons between the samples. The data are expressed as the mean \pm standard deviation (SD). Statistical significance was determined using the Student's t-test and ANOVA; the differences were considered significant at a P-value of $<0.05$.

\section{Results}

Establishment of the Cox-2 shRNA colorectal cancer cell line. To establish a stable Cox-2 knockdown colorectal cancer cell line, we developed three Cox-2 shRNA lentiviral vectors (pLenti6/Cox-2 shRNA1, pLenti6/Cox-2 shRNA2 and pLenti6/ Cox-2 shRNA3) and a control shRNA lentiviral vector. The pLenti6/Cox-2 shRNA vector and the ViraPower packaging mix were co-transfected, and the lentivirus in the supernatant was collected and concentrated 48 h later. The SW480/ EGFP cells were transduced with pLenti6/Cox-2 shRNA virus for $18 \mathrm{~h}$ and then selected with blasticidin for $48 \mathrm{~h}$. Ten days later, the cells were analyzed for Cox-2 expression using real-time PCR and western blot analysis. The real-time PCR and western blot analysis revealed that the pLenti6/Cox-2 shRNA 3 lentiviral vector was the most effective at blocking Cox-2 expression. Subsequently, we transduced the pLenti6/ 


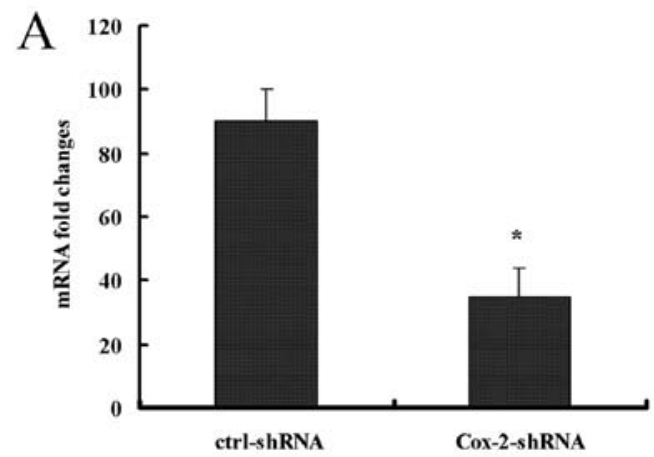

Ctrl-shRNA Cox-2-shRNA
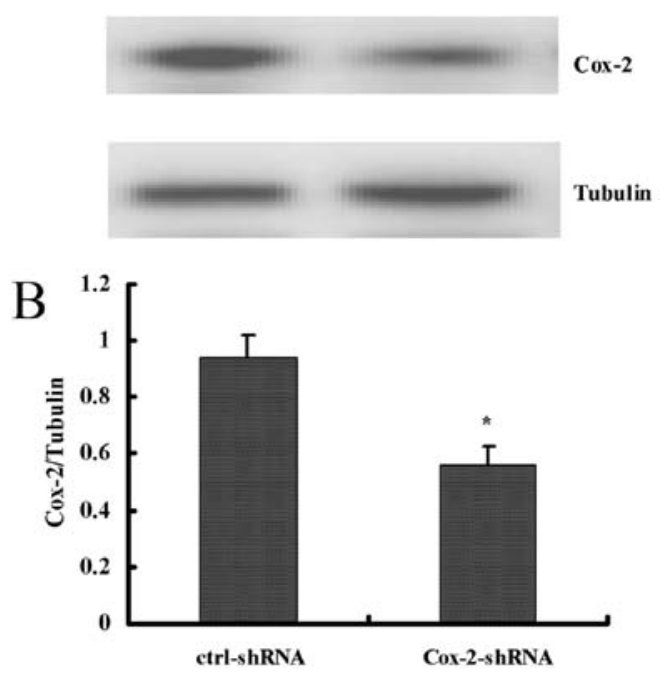

Figure 1 . The expression of Cox-2 was specifically knocked down by lentiviral vector-mediated RNA interference in SW480-EGFP colorectal cancer cells. (A) The Cox-2 mRNA expression was measured with real-time qPCR in the SW480-EGFP-Cox-2shRNA cells and in the SW480-EGFP-control cells; the Cox-2 mRNA level was markedly downregulated in the SW480EGFP-Cox-2shRNA cells compared with the SW480-EGFP-control cells. (B) The Cox-2 protein expression was measured by western blotting in the SW480-EGFP-Cox-2 shRNA cells and the SW480-EGFP-control cells. The Cox-2 protein level was markedly downregulated in the SW480-EGFP-Cox-2 shRNA cells compared with the SW480-EGFP-control cells. The results shown represent three separate experiments, and the data represent the mean $\pm \mathrm{SD}$. ${ }^{*} \mathrm{P}<0.05$

Cox-2 shRNA 3 into the SW480/EGFP cells and established blasticidin-resistant Cox-2 knockdown SW480-EGFP-Cox-2shRNA clones. The SW480-EGFP-Cox-2-shRNA clones were expanded and examined using real-time PCR, western blotting and immunocytochemical staining. The real-time PCR and western blot analysis revealed that there were no changes in Cox- 2 mRNA and protein expression in the control lentivirusinfected cell clone. Compared with the control lentivirus infected cell clone, the Cox- 2 mRNA and protein expression in the SW480-EGFP-Cox-2-shRNA cells were reduced by 71.2 and $47.2 \%$, respectively (Fig. 1).

Knockdown of the Cox-2 gene suppresses cell proliferation in vitro. The effect of Cox-2 protein reduction on the proliferation of colorectal cancer cells was analyzed using the MTT and plate clone formation assays. The MTT assay showed that the SW480-EGFP-Cox-2-shRNA had reduced growth ability compared with that of the control cells (Fig. 2A). The colony
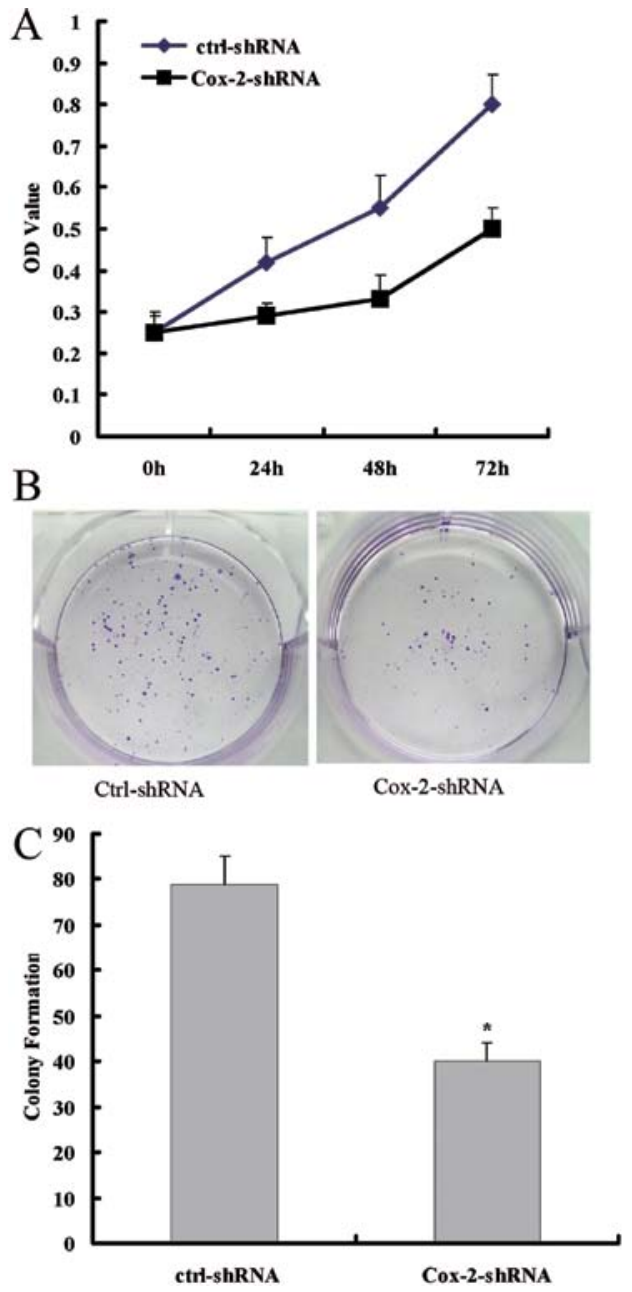

Figure 2. Knockdown of the Cox-2 gene suppresses cell proliferation in vitro (A) The growth of the SW480-EGFP-Cox-2 shRNA cells and SW480-EGFPcontrol cells was examined using an MTT assay over a 72-h period. The results shown represent three separate experiments, and the data represent the mean $\pm \mathrm{SD}$. ${ }^{*} \mathrm{P}<0.05$ compared to control-shRNA cells. (B and C) The growth of the SW480-EGFP-Cox-2 shRNA cells and SW480-EGFP-control cells was examined using the plate colony formation assay. The data shown represent the mean $\pm \mathrm{SD}$. ${ }^{*} \mathrm{P}<0.05$ compared to control-shRNA cells.

formation assay showed that the ability to form colonies of SW480-EGFP-Cox-2-shRNA cells was significantly reduced compared with that of the control cells (Fig. 2B and C).

Knockdown of the Cox-2 gene suppresses cell invasion in vitro. To measure the effect of Cox-2 gene knockdown on cell invasion, a matrigel invasion assay was performed. Compared with the control cells, the SW480-EGFP-Cox-2-shRNA cells displayed decreased invasion $(\mathrm{P}<0.05)$ (Fig. 3). These results demonstrated that the invasive ability of colorectal cancer cells was correlated with Cox-2 expression and that the Cox-2 gene knockdown effectively attenuates the invasion of colorectal cancer cells.

Cox-2 gene knockdown reduces the metastatic tumor formation. To evaluate the effect of the Cox-2 gene knockdown on colorectal cancer metastasis, an in vivo metastasis assay of the surgical orthotopic implantation of colorectal cancer cells was performed. The whole-body optical images were used to observe the real-time primary tumor growth and the 

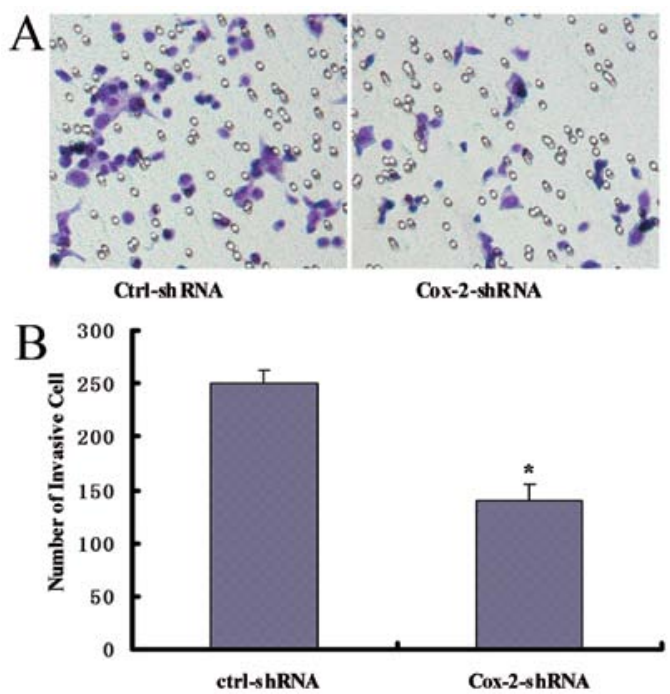

Figure 3. Knockdown of the Cox-2 gene suppresses cell invasion in vitro. The cell invasion capabilities of the SW480-EGFP-Cox-2shRNA cells and SW480-EGFP-control cells were examined using the transwell assay. The data shown represent the mean $\pm \mathrm{SD}$. ${ }^{*} \mathrm{P}<0.05$ compared to control-shRNA cells.

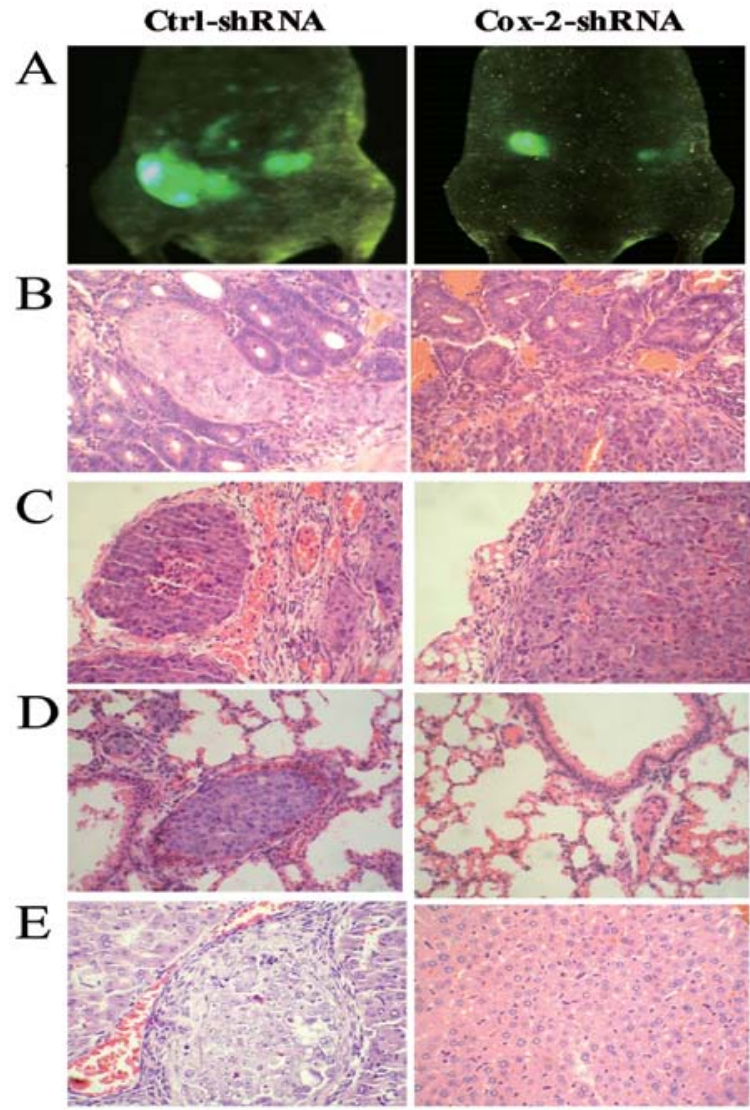

Figure 4. Knockdown Cox-2 gene suppresses cell metastasis in vivo. The metastastic ability of the SW480-EGFP-Cox- 2 shRNA cells and the SW480EGFP-control cells were examined using an orthotopic xenograft model in nude mice. (A) The whole-body fluorescence images of SW480-EGFP-Cox-2 shRNA cells and SW480-EGFP-control cells in the orthotopic xenograft model in nude mice. (B) The histological photomicrographs of the orthotopic xenograft tumor tissue sections stained with H\&E. (C) The histological photomicrographs of the metastasis into the celiac lymph node tissue sections stained with H\&E. (D) The histological photomicrographs of lung tissue sections stained with H\&E. (E) The histological photomicrographs of liver tissue sections stained with $\mathrm{H} \& \mathrm{E}$.

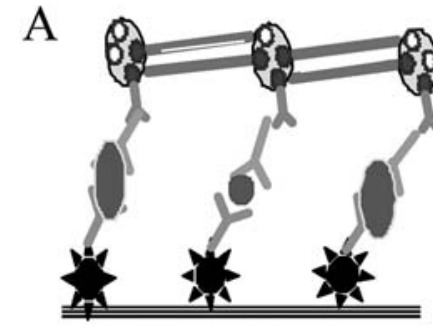

reporter DNA-protein conjugates

antibody

antigen

PCR

Immuno-bead

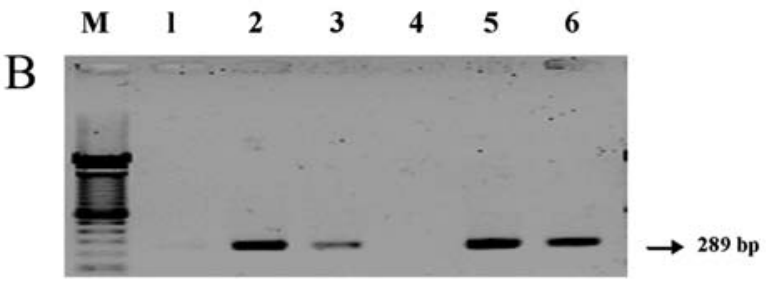

Figure 5. Serum Cox-2 can be used as a metastasis marker of colorectal cancer. (A) A schematic representation of the immuno-PCR assay. (B) The detection of the Cox- 2 protein from serum samples isolated from the colorectal cancer models. The agarose gel electrophoresis of the PCR amplicons reporter DNA produced by the immuno-PCR assay using serum samples from the colorectal cancer models. The PCR products were obtained from $5 \mu 1$ of serum. Lane 1, negative control; lane 2, positive control; lanes 3 and 5, control group; lanes 4 and 6, Cox-2 knockdown.

formation of metastatic lesions. Two months after the surgical orthotopic implantation of colorectal cancer cells into the nude mouse colon, the fluorescent images were used to compare the formation of metastatic lesions. The results showed that there were fewer metastatic lesions formed in the Cox-2-shRNA group compared with the control group. A high number of fluorescence signals was observed on the peritoneum and abdominal organs using the whole-body optical imaging system (Fig. 4A). The mice were sacrificed and autopsied following blood collection. The incidence of metastasis in the liver, lungs, or other organs was determined by macroscopic and histological examinations. In the control group, $87.5 \%$ of the mice developed peritoneal metastases; however, in the SW480-Cox-2shRNA group, only $37.5 \%$ of the animals exhibited peritoneal metastases. The incidence of hepatic metastasis and lung lesions in the mice from the control group was $37.5 \%$ (3 of 8 ) and $12.5 \%$ (1 of 8), respectively. The SW480Cox-2shRNA group did not produce detectable tumors in the liver or other organs (Fig. 4B-E). These results indicate that Cox-2 silencing was sufficient to decrease the metastasis of colorectal cancer cells.

Cox-2 gene knockdown reduces the positive detection incidences of Cox-2 protein in the serum of an imageable metastasis model of colorectal cancer. To detect very low concentrations of serum Cox-2 protein, an improved immunobead PCR protocol was established and utilized. Our data show that the 289 bp amplified DNA bands were found in the positive control, and no amplified DNA band was seen in the negative control (Fig. 5B). The positive detection rate for the Cox-2 protein in the SW480-shRNA group and SW480-CtrlshRNA group was 12.5 and $87.5 \%$, respectively. The positive detection rate for the Cox-2 protein in the samples from the SW480-shRNA group was significantly lower than that in the 
SW480-Ctrl-shRNA group $(\mathrm{P}<0.05)$. These results suggest that the Cox-2 protein can be detected in the serum of a metastatic colorectal cancer nude mouse model and that Cox-2 can be used as a colorectal cancer metastasis marker.

\section{Discussion}

Cox-2 is an inducible enzyme that converts arachidonic acid to prostaglandins. Cox-2 catalyzes the oxidation of arachidonic acid, which produces prostaglandins that may accelerate the carcinogenesis and metastasis process. It is possible that the increased levels of Cox-2 serve to lower the intracellular level of free arachidonic acid and, thereby, prevent apoptosis by the depletion of the apoptotic signal (20). Data have shown that the overexpression of Cox-2 is related to colorectal cancer metastasis (12). Our data also show that the expression of Cox-2 is related to colorectal cancer metastasis. Additionally, we found that Cox-2 can be overexpressed in the glandular tube of colorectal cancer and in the surrounding inflammatory tissue (11). Because Cox-2 can be secreted into the glandular tube of colorectal cancer and the surrounding inflammatory tissue, whether it can be detected in the serum has not been determined.

In previous studies of azoxymethane-induced colon carcinogenesis in rats, treatment with a selective Cox-2 inhibitor (NS-398) has been shown to reduce the tumor size and multiplicity $(21,22)$. The clinical relevance of these findings was shown in patients with germ line mutations in the APC gene and the autosomal dominant inherited syndrome of familial adenomatous polyposis. In these patients, treatment with the NSAID has been shown to regress existing colorectal adenomas. Furthermore, the NSAID treatments aimed at inhibiting Cox-2 have been proven to be chemopreventive, reducing both the polyp number and the polyp burden following clinical trials in colon cancer patients (23). The side-effects of some of the Cox-2 inhibitors that have emerged following long-term treatment have caused much concern.

The use of RNA interference (RNAi) technology to reduce gene expression has been become widely utilized. RNA interference (RNAi) is a gene regulatory system in which small RNA molecules silence genes that have a similar sequence to the small RNAs. Short-hairpin RNA has been widely used to reduce the expression of many target genes due to their high specificity and apparent non-toxicity (24-27). The published data show that a reduction in the expression of Cox- 2 in a colorectal cancer cell line by RNA interference (RNAi) technology resulted in decreased cell proliferation, invasion and metastasis (28); these data were obtained using a cell model and not from suitable animal model. Additionally, the previous experiments used a routine transfect reagent to transiently transfect shRNA vector plasmid into the cells and did not generate a stable Cox-2 gene knockdown cell line. In the current study, we used a lentiviral vector-based RNAi expression system in which the expression of shRNAs is driven by a U6 promoter to stably knockdown the expression of Cox-2 mRNA in the colorectal cancer SW480-EGFP cell line. The lentiviral expression system offers several advantages over other transfection reagents. The lentiviral system can easily infect over $90 \%$ of cultured cells, which is sufficient for studying the effects of RNAi on endogenous gene expression
(29). Lentivirus is relatively easy to generate, and thus, it has been applied to large-scale, high-throughput RNAi assays for studying gene functions (30). Finally, the lentiviral system has the advantage of the long-term stable expression of a transgene to establish stable transfected cell lines (31). Using this system, we generated a Cox-2 gene stable knockdown colorectal cell line, the SW480-EGFP-Cox-2-shRNA cell line, and used this cell line to generate an imageable metastasis model of colorectal cancer.

In this study, we found that the knockdown of Cox-2 in the SW480-EGFP-Cox-2shRNA cells abrogated the cell's ability to proliferate and invade and strongly impaired colon xenograft formation. We found that the knockdown of Cox-2 expression appeared to have an inverse correlation with tumorigenesis. The experiments also demonstrate that a reduction in Cox-2 expression in colorectal cancer cells decreased angiogenesis. The whole-body optical imaging system allows for the continuous visual monitoring of malignant tumor growth within intact animals. Our data show that the reduced expression of Cox-2 in SW480-EGFPCox-2shRNA cells abrogated their ability to metastasize to lymph nodes, lungs or liver. In the orthotopic xenograft model, the data showed that the reduced expression of Cox-2 in the SW480-EGFP-Cox-2shRNA cells abrogated their ability to develop lung and hepatic metastases. These results support the concept that Cox-2 may be a positive regulator of tumor growth in colorectal cancer. A stable lentivirusbased Cox- 2 knockdown mediated by RNAi impaired the invasive and metastatic ability of colorectal cancer cells. Our study confirms that the Cox- 2 gene may be an effective target for cancer therapies. The endogenous Cox-2 gene can be specifically downregulated by RNAi in a colorectal cancer cell line. All of these data show that the inhibition of Cox-2 can reduce the risk of colorectal cancer development and metastasis. The results reported here indicate an easy yet powerful and highly selective lentivirus-based method to knockdown Cox-2 expression in a stable and long-lasting manner. Furthermore, we propose the possibility of an in vivo application of this anti-Cox-2 lentiviral vector to establish a stable human cancer metastasis model system.

Because Cox-2 is a key gene and pays an important role in colorectal cancer development and metastasis, it is frequently highly expressed in colorectal cancer. The ability of Cox-2 to infiltrate into the serum has not been investigated, and whether it can be used as a serum marker of colorectal cancer still needs to be explored. As we have found that Cox-2 can be detected in the glandular cavity of colorectal cancer and in the surrounding inflammatory tissue by immunohistochemistry, we speculated that the Cox-2 protein can be secreted into the interstitial liquid and then cycled into the blood at a very low concentration. Thus, in this study, we generated a new, improved immunobead-PCR method to detect very low concentrations of the tumor marker in serum samples.

Initially, we used Dynabeads ${ }^{\circledR}$ M-280 sheep anti-rabbit IgG immunobeads, rabbit anti-Cox-2 antibody and a mouse antiCox-2 antibody to capture low concentrations of Cox-2 protein in serum samples. The Dynabeads ${ }^{\circledR} \mathrm{M}-280$ sheep anti-rabbit $\mathrm{IgG}$ are uniform, superparamagnetic, polystyrene beads with affinity-purified sheep anti-rabbit IgG covalently bound to the bead surface. These polyclonal antibodies bind both heavy and 
light chain rabbit IgG. They are designed as a solid support for the simple and efficient binding of target molecules. These beads allow for the isolation and subsequent handling of target molecules in a highly specific manner. The beads are added directly to the sample containing the target antibody/antigen (Cox-2 antibody/Cox-2 protein). After a short incubation that allows for the affinity capture of the target, the beads are pulled to the side of the test tube by a magnet, thus allowing for the aspiration of the unbound material. The magnetic separation facilitates the easy washing and concentration of the isolated target molecule. The target molecule can be eluted off of the beads with conventional elution methods. Second, this assay utilizes the self-assembly capabilities of semi-synthetic DNA-protein conjugates $(32,33)$. In particular, the covalent ssDNA-STV conjugates are employed as molecular adapters for the effective DNA-directed immobilization of the captured antibodies on solid supports containing complementary oligonucleotides. The capture antibodies are used for the selective capture of the protein antigen, similar to the conventional sandwich ELISA. Subsequently, immuo-PCR is employed as a high-sensitivity detection method, taking advantage of the conjugates produced by the self-assembly of the STV, biotinylated dsDNA and antibodies directed against the protein. This assay allowed us to detect very low amounts of antigen, typically 100-1,000-fold less than is detectable by conventional sandwich ELISAs (34). In addition, this immunoassay can be performed in a single step by simultaneously tagging the protein with capture and detection reagents, thereby significantly reducing handling time. In our experiment, a false positive result occasionally occurred because of the sensitivity of this assay. Therefore, strict positive and negative controls are needed and the procedure should be performed carefully each time this sensitive assay is used. Using this improved immunobeads PCR assay, we found that the Cox-2 protein infiltrated into the serum. Additionally, we found that the positive detection incidence of the Cox-2 protein in samples from the SW480-shRNA group was significantly lower than that in the control group with metastasis.

Taken together, our results indicate that the knockdown of Cox- 2 expression by RNAi suppressed the proliferation of colorectal cancer both in vitro and in vivo. This study also demonstrated that targeting Cox- 2 in vivo reduced the metastatic potential of colorectal cancer cells. Thus, Cox-2 is a promising marker for the diagnosis of colorectal metastasis, and it is also a promising target for therapeutic intervention.

\section{Acknowledgements}

This study was supported by the major projects of the National Natural Science Foundation of China (no. 81090422/H1606), the National Natural Science Foundation of China (nos. 30770976, 81071735, 81172054), the National Basic Research Program of China (973 Program, no. 2010CB529403), the Science and technology projects in Guangdong Province (no. 2010B031500012), the Guangdong Provincial Key Science and Technology Innovation Fund for Higher Education (no. GXZD1016), the Innovative Research Team Foundation in University (no. IRT0731) and the Universities in Guangdong Province 211 key construction projects.

\section{References}

1. Masunaga R, Kohno H, Dhar DK, et al: Cyclooxygenase-2 expression correlates with tumor neovascularization and prognosis in human colorectal carcinoma patients. Clin Cancer Res 6: 4064-4068, 2000.

2. Wendum D, Masliah J, Trugnan G and Fléjou JF: Cyclooxygenase- 2 and its role in colorectal cancer development. Virchows Arch 445: 327-333, 2004.

3. Möbius C, Stein HJ, Spiess C, et al: COX2 expression, angiogenesis, proliferation and survival in Barrett's cancer. Eur J Surg Oncol 31: 755-759, 2005.

4. Wang Q, Takei Y, Kobayashi O, Osada T and Watanabe S: Cyclooxygenase 2 modulates killing of cytotoxic T lymphocytes by colon cancer cells. J Clin Biochem Nutr 45: 163-170, 2009.

5. Boland GP, Butt IS, Prasad R, Knox WF and Bundred NJ: Cox-2 expression is associated with an aggressive phenotype in ductal carcinoma in situ. Br J Cancer 90: 423-429, 2004.

6. Singh B, Berry JA, Shoher A, Ramakrishnan V and Lucci A: Cox-2 overexpression increases motility and invasion of breast cancer cells. Int J Oncol 26: 1393-1399, 2005.

7. Wang W, Bergh A and Damber JE: Cyclooxygenase-2 expression correlates with local chronic inflammation and tumor neovascularization in human prostate cancer. Clin Cancer Res 11: 3250-3256, 2005.

8. Petersen S, Haroske G, Hellmich G, Ludwig K, Petersen C and Eicheler W: Cox-2 expression in rectal carcinoma: immunohistochemical pattern and clinical outcome. Anticancer Res 22: 1225-1230, 2002.

9. Eberhart CE, Coffey RJ, Radhika A, Giardiello FM, Ferrenbach S and DuBois RN: Up-regulation of cyclooxygenase 2 gene expression in human colorectal adenomas and adenocarcinomas. Gastroenterology 107: 1183-1188, 1994.

10. Kim JY, Lim SJ and Park K: Cyclooxygenase-2 and c-erbB-2 expression in colorectal carcinoma assessed using tissue microarrays. Appl Immunohistochem Mol Morphol 12: 67-70, 2004.

11. Li ZG, Liu TF, Xie WB, Zhou J, Yu L and Ding YQ: Association of abnormal cyclooxygenase-2 gene expression with colorectal carcinoma metastasis. Nan Fang Yi Ke Da Xue Xue Bao 26: 1408-1411, 2006 (In Chinese).

12. Dempke W, Rie C, Grothey A and Schmoll HJ: Cyclooxygenase-2: a novel target for cancer chemotherapy. J Cancer Res Clin Oncol 127: 411-417, 2001.

13. Narayanan BA, Narayanan NK, Pttman B and Reddy BS: Adenocarcina of the mouse prostate growth inhibition by celecoxib: downregulation of transcription factors involved in COX-2 inhibition. Prostate 66: 257-265, 2006.

14. Yao M,Kargman S,Lam EC, et al: Inhibition of cyclooxygenase-2 by rofecoxib attenuates the growth and metastatic potential of colorectal carcinoma in mice. Cancer Res 63: 586-592, 2003.

15. Bottone FG Jr, Martinez JM, Collins JB, Afshari CA and Eling TE: Gene modulation by the cyclooxygenase inhibitor, sulindac sulfide, in human colorectal carcinoma cells: possible link to apoptosis. J Biol Chem 278: 25790-25801, 2003.

16. Dubé C, Rostom A, Lewin G, et al: The use of aspirin for primary prevention of colorectal cancer: a systematic review prepared for the U.S. Preventive Services Task Force. Ann Intern Med 146: 365-375, 2007.

17. Chan AT, Ogino S and Fuchs CS: Aspirin and the risk of colorectal cancer in relation to the expression of Cox-2. N Engl J Med 356: 2131-2142, 2007.

18. Oshima M, Murai N, Kargman S, et al: Chemoprevention of intestinal polyposis in the Apcdelta716 mouse by rofecoxib, a specific cyclooxygenase-2 inhibitor. Cancer Res 61: 1733-1740, 2001.

19. Rashidi B, Gamagami R, Sasson A, Sun FX, Geller J, Moossa AR and Hoffman RM: An orthotopic mouse model of remetastasis of human colon cancer liver metastasis. Clin Cancer Res 6: 2556-2561, 2000.

20. Cao Y, Pearman AT, Zimmerman GA, McIntyre TM and Prescott SM: Intracellular unesterified arachidonic acid signals apoptosis. Proc Natl Acad Sci USA 97: 11280-11285, 2000.

21. Yoshimi N, Kawabata K, Hara A, Matsunaga K, Yamada Y and Mori H: Inhibitory effect of NS-398, a selective cyclooxygenase-2 inhibitor, on azoxymethane-induced aberrant crypt foci in colon carcinogenesis of F344 rats. Jpn J Cancer Res 88: 1044-1051, 1997. 
22. Yoshimi N, Shimizu M, Matsunaga K, Yamada Y,Fujii K, Hara A and Mori H: Chemopreventive effect of N-(2-cyclohexyloxy4-nitrophenyl)methane sulfonamide (NS-398), a selective cyclooxygenase- 2 inhibitor, in rat colon carcinogenesis induced by azoxymethane. Jpn J Cancer Res 90: 406-412, 1999.

23. Steinbach G, Lynch PM, Phillips RK, et al: The effect of celecoxib, a cyclooxygenase- 2 inhibitor, in familial adenomatous polyposis. N Engl J Med 342: 1946-1952, 2000.

24. Caplen NJ, Parrish S, Imani F, Fire A and Morgan RA: Specific inhibition of gene expression by small double-stranded RNAs in invertebrate and vertebrate systems. Proc Natl Acad Sci USA 98: 9742-9747, 2001.

25. Elbashir SM, Harborth J, Lendeckel W, Yalcin A, Weber K and Tuschl T: Duplexes of 21-nucleotide RNAs mediate RNA interference in cultured mammalian cells. Nature 411: 494-498, 2001.

26. Soutschek J, Akinc A, Bramlage B, Charisse K, et al: Therapeutic silencing of an endogenous gene by systemic administration of modified siRNAs. Nature 432: 173-178, 2004.

27. Lu PY, Xie F and Woodle MC: In vivo application of RNA interference: from functional genomics to therapeutics. Adv Genet 54: 117-142, 2005.

28. Charames GS and Bapat B: Cyclooxygenase-2 knockdown by RNA interference in colon cancer. Int J Oncol 28: 543-549, 2006.
29. Bartosch B and Cosset FL: Strategies for retargeted gene delivery using vectors derived from lentiviruses. Curr Gene Ther 4: 427-443, 2004

30. Bailey SN, Ali SM, Carpenter AE, Higgins CO and Sabatini DM: Microarrays of lentiviruses for gene function screens in immortalized and primary cells. Nat Methods 3: 117-122, 2006

31. Abbas-Terki T, Blanco-Bose W, Déglon N, Pralong W and Aebischer P: Lentiviral-mediated RNA interference. Hum Gene Ther 13: 2197-201, 2002.

32. Niemeyer CM, Wacker R and Adler M: Combination of DNA-directed immobilization and immuno-PCR: very sensitive antigen detection by means of self-assembled DNA-protein conjugates. Nucleic Acids Res 31: e90, 2003.

33. Grinde B, Jonassen $\mathrm{T}$ and Ushijima $\mathrm{H}$ : Sensitive detection of group A rotaviruses by immunomagnetic separation and reverse transcription-polymerase chain reaction. J Virol Methods 55: 327-338, 1995.

34. Xiang CQ, Shen CL, Wu ZR, Qin YQ, Zhang YY, Liu CZ, Chen JG and Zhang SN: Detection of mutant p53 protein in workers occupationally exposed to benzidine. J Occup Health 49: 279-284, 2007 